\title{
Pharmaciana
}

Vol.11, No.2, July 2021, Page. 239-250

ISSN: 2088 4559; e-ISSN: 24770256

DOI: $10.12928 /$ pharmaciana.v11i2.21044

\section{Enhanced skin localization of doxycycline using microparticles and hydrogel: effect of oleic acid as penetration enhancer}

\author{
Aliyah Aliyah*, Windy Winalda Oktaviani , Arini Putri Erdiana, \\ Kadek Saka Dwipayanti, Rifka Nurul Utami , Andi Dian Permana \\ Department of Pharmaceutics, Faculty of Pharmacy, Hasanuddin University, Makassar, Indonesia \\ Jl. Perintis Kemerdekaan KM 10, Makassar, Indonesia
}

Submitted: 05-07-2021

Reviewed: 12-07-2021

Accepted: 27-07-2021

\begin{abstract}
One of the disadvantages of gel preparations is the poor ability to penetrate the skin. Herein we developed a sodium carboxymethylcellulose (NaCMC)-based gel containing microparticle of doxycycline hyclate (DOX). Oleic acid (OA) was added into the gel formulation specifically to increase the penetrability of DOX microparticles. The objective of this work was to determine the effect of varying concentration of $\mathrm{OA}$ on the physical characteristics, penetration and retention abilities of DOX. DOX microparticles were initially prepared using PLGA as a matrix and then incorporated into a NaCMC-based gel with various OA concentrations: $0 \%, 2.5 \%, 5 \%, 7.5 \%$, and $10 \%$ for F1, F2, F3, F4, and F5, respectively. The gel preparations were evaluated for their organoleptic test, homogeneity, $\mathrm{pH}$ measurement, viscosity, spreadability, as well as ex vivo penetration and retention abilities. The physical characteristics tests revealed a homogeneous yellow gel with a distinctive odor and $\mathrm{pH}$ values compatible with the $\mathrm{pH}$ requirements of human skin. The retention test showed the formulation retained $1236.46 \mu \mathrm{g}$ of DOX in the skin. Finally, the skin retention of DOX from microparticles loaded gel was significantly higher compared to the free DOX loaded gel, indicating the microparticles can be extremely effective in retaining the DOX in the infected area.
\end{abstract}

Keywords: oleic, acid, gel, doxycycline microparticle, penetration enhancer

\footnotetext{
*Corresponding author:

Aliyah Aliyah

Faculty of Pharmacy, Hasanuddin University

J1. Perintis Kemerdekaan KM. 10, Makassar, Indonesia

Email: aliyahputranto@yahoo.co.id
} 


\section{INTRODUCTION}

Skin provides a physical protective barrier to the body against infections, the term of diseases caused by entry and development of microorganisms such as bacteria, fungi, parasites and viruses, which damage the skin thereby causing various clinical symptoms and signs (Chiller et al., 2001). Staphylococcus aureus is the most common bacteria causing chronic wound and soft tissue infections (Hatlen and Miller, 2021) . Infection is triggered by the presence of microorganisms that can penetrate the deeper skin layers of the dermis or subcutaneous tissue thereby damaging the tissue and impairing immune or healing response (Kalan and Femling, 2017).

Doxycycline hyclate (DOX) is one of the antibiotics commonly used to treat skin infections (Smith et al., 2020). Doxycycline hyclate is reversibly bound to the 30S ribosomal subunits and prevents the formulation of peptide chains of amino acids, thereby inhibiting protein synthesis, with a broad spectrum of antibacterial properties and effective against $P$. aeruginosa and $S$. aureus (Chukwudi, 2016). According to (Borse et al., 2020), doxycycline hyclate showed an inhibition zone between $13.66 \pm 1.69 \mathrm{~mm}$ to $32.00 \pm 1.63 \mathrm{~mm}$ against $S$. aureus.

Oral administration of DOX can cause side effects such as abdominal pain, gastrointestinal irritation and other side effects, whereas topical application can be more beneficial for the treatment and healing of bacterial wound infections (Smith et al., 2020). Topical antibiotics can minimize the side effects and local administration may hinder the potential for systemic absorption of antibiotics. This will subsequently reduce antibiotic resistance, act more selectively against the source of infection, and avoid first-pass metabolism (Marco et al., 2020). However, topical administration of a drug is usually limited to certain drug molecules, a factor which can hinder absorption of the drug by the infected skin (Kim et al.,2019). Stratum corneum, located in the outermost of epidermis, is considered to be the major barrier of drug absorption through the skin (Permana et al., 2020). Gels are one of the most commonly used topical preparations for use in treating skin infections. Gels can provide several advantages, as their easy formulation is relatively easy compared to other semisolid preparations, they are biocompatible, have a high skin retention time, high spreadability and a cooling effect on the application site (Nandini, 2009).

However, the main drawback of gel preparation is poor drug penetration through the skin (Prakash et al., 2016) Hence, the addition of a skin penetration enhancer such as oleic acid is often considered necessary. Oleic acid is a mono-unsaturated fatty acid reported to increase the penetration of drugs through the skin. This compound acts by disrupting the densely arranged intercellular lipid domain of stratum corneum (Lengyel et al., 2019). However, when it reaches the dermal layer, DOX has a low retention ability, which means that doxycycline can quickly leave the dermis and enter the systemic circulation (Permana et al., 2020). For that reason, it is necessary to develop a drug delivery system and formulation to increase drug retention at the infection site and avoid systemic circulation.

The use of microparticles is one option which has been studied as a drug delivery carrier for controlled release (Campos et al., 2013). Microparticles can be defined as particles possessing spherical shapes with sizes between $1 \mu \mathrm{m}$ and $1000 \mu \mathrm{m}$, containing a mixture of active compounds and raw materials consisting of coatings attached to a matrix structure (Prow et al., 2011). It has been shown that the use of PLGA (poly lactic-co-glycolic acid) polymers can retain specific antimicrobial agents at the infected site, thereby reducing unwanted side effects leading to systemic issues. PLGA has several advantages including suitable degradation under physiological conditions and sustained drug release (Machado et al., 2013; Mazzara et al., 2019). Gel preparations contain gel bases which can be hydrophilic or hydrophobic (Patil et al., 2019). Carboxymethylcellulose sodium (NaCMC) is one of the gelling agents used in gel preparations at concentrations of 1-5\%; it is a water-soluble cellulose derivative with good bioadhesive, biodegradability, biocompatibility and non-toxic properties (Akalin and Pulat, 2018). NaCMC is a polyelectrolyte that has varied ionic strength, sensitivity to $\mathrm{pH}$, and can easily form a gel due to the presence of hydroxyl groups (Lopez et al., 2018). This study aimed to formulate PLGA microparticles containing DOX with variations in the concentration of $\mathrm{OA}$ as a penetration enhancer in gel preparations. Following characterization of the 
gel formulation, ex vivo permeation and retention trials were carried out to evaluate the ability of this approach to deliver DOX to the desired area.

\section{MATERIALS AND METHODS}

\section{Chemicals and materials}

Doxycycline hyclate (DOX) (purity, $\geq 98 \%$ ) was obtained from Alfa Aesar (UK). Sodium carboxymethylcellulose (NaCMC), PVA $(9-10 \mathrm{kDa})$ and oleic acid (OA) were obtained from SigmaAldrich (Singapore). All other reagents used were analytical grade.

\section{Formulation of DOX-loaded microparticles}

The incorporation of DOX into microparticle formulations was performed using double emulsion (W/O/W) solvent evaporation technique (Soomherun et al., 2017). Firstly, $250 \mathrm{mg}$ of DOX was dissolved in $10 \mathrm{~mL}$ of distilled water. This solution was emulsified in a PLGA solution $(500 \mathrm{mg}$ in $20 \mathrm{~mL}$ of dichloromethane) using a homogenizer at 3,000 rpm for 5 minutes, forming a W/O emulsion. This mixture was then emulsified in $50 \mathrm{~mL}$ PVA $(9-10 \mathrm{kDa})$ solution in water $(2 \% \mathrm{w} / \mathrm{v})$ using a homogenizer at 3,000 rpm for 10 minutes, creating a W/O/W emulsion. In order to evaporate the solvent and form the microparticles, the double emulsion was stirred at room temperature for $8 \mathrm{~h}$. The microparticles were finally collected following three washing steps with distilled water using centrifugation at 2,000 rpm for $15 \mathrm{~min}$ to remove the unencapsulated DOX and PVA.

The particle size and polydispersity index of the microparticles were observed using a light microscope. The encapsulation efficiency (EE) of DOX in the microparticles was determined through an indirect technique (Soomherun et al., 2017). After the first centrifugation in the washing steps, the supernatant containing free DOX was subjected to UV-Visible spectrophotometry. The EE of DOX in the microparticles was determined using equation (1).

$$
\mathrm{EE}(\%)=\frac{\text { DoX }_{\text {total }}-\text { Dox }_{\text {fras }}}{\text { DoX }_{\text {total }}} \times 100 \%
$$

\section{Preparation of gel containing DOX microparticles}

Gels were prepared using $\mathrm{NaCMC}$ and oleic acid as a gelling agent and as a penetration enhancer, respectively. Additionally, glycerin was used as a humectant and DMDM hydantion was used as preservative. The compositions of each formulation are shown in Table 1. NaCMC aliquots were weighed accurately and dissolved in warm water (temperature $70^{\circ} \mathrm{C}$ ) in a beaker, then homogenized at 2,000 rpm for 10 minutes until a colloidal solution was formed. Glycerin was added to the solution, then DOX microparticles were added to the solution which was then stirred. Once the mix was homogeneous, OA was mixed into the solution and DMDM hydantoin was added to the solution which was then stirred and homogenized at $1,000 \mathrm{rpm}$ for 15 minutes to form a gel.

Table 1. Composition of gel formulations containing DOX microparticles (\%w/w)

\begin{tabular}{ccccccc}
\hline Composition & $\begin{array}{c}\text { DOX } \\
\text { Microparticles }\end{array}$ & NaCMC & Glycerin & Oleic acid & $\begin{array}{c}\text { DMDM } \\
\text { Hydantoin }\end{array}$ & Water \\
\hline F1 & 2.5 & 5 & 10 & - & 0.1 & until 100 \\
F2 & 2.5 & 5 & 10 & 2.5 & 0.1 & until 100 \\
F3 & 2.5 & 5 & 10 & 5 & 0.1 & until 100 \\
F4 & 2.5 & 5 & 10 & 7.5 & 0.1 & until 100 \\
F5 & 2.5 & 5 & 10 & 10 & 0.1 & until 100 \\
\hline
\end{tabular}




\section{Gel characterization}

\section{Organoleptic test}

Organoleptic observations were carried out by visually or directly observing the color, smell, and appearance of the gel preparations (Ardana et al., 2015).

\section{Homogeneity test}

The homogeneity test was carried out by applying the gel preparation to a piece of glass or other suitable transparent material, then observing the homogeneity of the preparation (Borse et al., 2020).

\section{pH measurement}

The $\mathrm{pH}$ measurement of the resulting gel preparations was performed using a calibrated digital $\mathrm{pH}$ meter. The procedure was repeated three times and the $\mathrm{pH}$ meter readings were recorded. Results were compared to the target $\mathrm{pH}$ for skin application, which is 4.5-6.5 (Borse et al., 2020).

\section{Viscosity test}

The viscosity of the gel preparations was measured using a Brookfield Viscometer by dipping the Spindle 6 into the gel preparation at a speed of $50 \mathrm{rpm}$. The procedure was repeated three times for each preparation and the mean viscosity was recorded in centipoise units (cPs) (Permana et al., 2020).

\section{Spreadability test}

Gel spreadability was measured by placing 1 gram of gel preparation between two glass plates measuring $20 \times 20 \mathrm{~cm}$, then pressing them together horizontally. Weights were then placed on top of the glass plates for 1 minute each, with a sequence of $125,225,325,425$ and $525 \mathrm{~g}$. The diameter of the gel dispersion was measured using a caliper (precision $0.15 \mathrm{~mm}$ ). The procedure was repeated three times for each preparation and the results obtained were the mean of these three replicates (Permana et al., 2020).

\section{In vitro skin occlusivity}

Investigation of in vitro skin occlusivity was performed according to (Cohen-Sela et al., 2009). Initially, $250 \mathrm{mg}$ of each gel formulation was weighed and applied to a Whatman $2.5 \mu \mathrm{m}$ filter paper. Water $(50 \mathrm{~mL})$ was placed in a $100 \mathrm{~mL}$ beaker and covered with the filter paper containing the gel formulation. A filter paper without formulation was utilized as a control. The beakers were placed in an oven at $32^{\circ} \mathrm{C}$. At predetermined times $(0,6,24$ and $48 \mathrm{~h})$, the weight of the system was calculated. Finally, the occulisivity $\left(\mathrm{F}_{0}\right)$ was calculated using the following equation (2):

$$
\mathrm{F}_{0}=\frac{W 0-W 1}{W 0} \times 100 \%
$$

where, $W O$ is the water loss of the control and $W 1$ is the water loss of the gel formulation

Effect of gel formulation on microparticles size and PdI

After dispersing the gel preparations in distilled water, the mixture was centrifuged at 2,000 rpm for 15 minutes and washed three times with water. The pellets were resuspended with water and then microparticle size and PDI were calculated.

\section{Ex vivo dermal delivery}

The gel preparations were evaluated for their ex vivo dermal delivery of the gel using a Franz diffusion cell. In this study, phosphate buffer saline (PBS) ( $\mathrm{pH}$ 7.4) was used as the medium. Initially, rat abdominal skin was dissected, and the remaining hairs were trimmed. Then, prior to each experiment, skins were soaked in PBS. PBS was filled into the receptor section, and the skin was attached in Franz diffusion cell. The evaluation was conducted at $37 \pm 0.5{ }^{\circ} \mathrm{C}$ with constant stirring at $100 \mathrm{rpm}$. An aliquot of 1 gram of gel (equivalent to $10 \mathrm{mg}$ of DOX) was spread uniformly on the 
surface of skin in the donor compartment. Samples were taken $(1.5 \mathrm{~mL})$ at various intervals in time, and the volume taken was replaced with the same volume of PBS pH 7.4 at the same temperature. Sample content was examined through UV-Visible spectrophotometry, the drug concentration in the sample was determined using a standard curve, then the rate of release (penetration flux) of the preparation was calculated. The penetration flux ( $\mathrm{J})$ was calculated using following equation (3):

$$
\mathrm{J}=\frac{M}{S P t}
$$

where, $M$ is the cumulative total of sample passing through the membrane $(\mu \mathrm{g}), S$ is diffusion area width $(\mathrm{cm})$, and $T$ is time (h)

\section{Retention test}

The rat skin used in the permeation test was placed in $50 \mathrm{~mL}$ of PBS pH 7.4 solution and the mixture was homogenized at $1,000 \mathrm{rpm}$ for 15 minutes. The supernatant was collected and centrifuged for 30 minutes at a speed of $5,000 \mathrm{rpm}$, then the absorbance was determined through spectrophotometer.

\section{Statistical analysis}

All results were reported as mean \pm standard deviation $(\mathrm{SD})$ of the mean. Data were tabulated (including mean and SD calculations) in Microsoft ${ }^{\circledR}$ Excel ${ }^{\circledR} 2013$ (Microsoft Corporation, Redmond, USA). Statistical analyses were implemented in GraphPad Prism® version 5 (GraphPad Software, San Diego, California, USA). In all cases, significance was determined at the $95 \%$ confidence level ( $p<$ $0.05)$.

\section{RESULT AND DISCUSSION}

\section{Formulation of DOX-loaded microparticles}

PLGA microparticles containing DOX were formulated using a multiple emulsion technique. This technique is suitable for loading hydrophilic drugs, like DOX, into hydrophobic PLGA microparticles (Cohen-Sela et al., 2009). Microparticle diameter was $2.98 \pm 0.21 \mu \mathrm{m}$ with a PDI value of $0.281 \pm 0.03$. With respect to the EE value, due to hydrophilicity, approximately $40.32 \pm 0.04 \%$ DOX was encapsulated in the PLGA microparticles. Considering the EE values of DOX, to achieve a concentration of $1 \% \mathrm{w} / \mathrm{v}$ DOX in the gel formulation, $2.5 \%$ DOX microparticles were incorporated into the NaCMC-based gel formulation with various OA concentrations: F1 (0\%), F2 (2.5\%), F3 (5\%), F4 (7.5\%) and F5 (10\%).

\section{Gel characteristics \\ Organoleptic and homogeneity}

Based on organoleptic test results presented in Figure 1a, all tested formulations had similar physical characteristics. All the formulations were colorless to yellowish white, and had a distinctive smell. The homogeneity evaluation (Figure 1b) showed that all gel preparations had good homogeneity with none exhibiting grittiness or coarse granules. Based on these results, all formulations were evaluated further. 
(a)
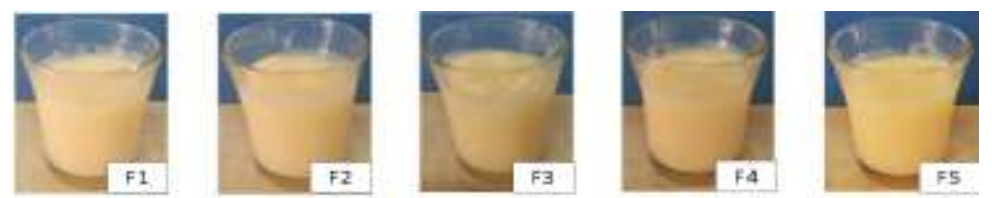

(b)
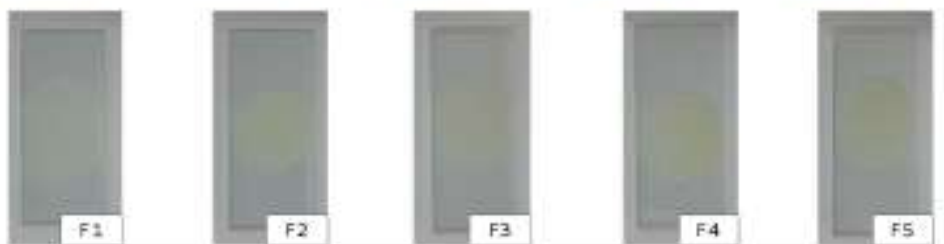

Figure 1 (a) Physical aspect of doxycycline microparticle gel preparations with various oleic acid concentrations: F1 (0\%), F2 (2.5\%), F3 (5\%), F4 (7.5\%) and F5 (10\%); (b) Homogeneity tests results for the doxycyline microparticle gel preprations

\section{pH and viscosity}

The $\mathrm{pH}$ of the gel preparations was measured as it is crucial to develop to develop formulations with specific $\mathrm{pH}$ values appropriate for topical preparations to avoid irritation of the skin (Wohlrab and Gebert, 2018). The $\mathrm{pH}$ values of the DOX microparticle gels ranged from 5.51 to 5.91 (Table 1). The widely accepted ideal $\mathrm{pH}$ range for skin treatments is $\mathrm{pH} 4.5$ - 6.5 (Ali and Yosipovitch, 2013). These results indicate that all formulations met the gel skin criteria requirement, and would be unlikely to produce skin irritation.

Viscosity is a descriptor of preparations which influences the characteristics of the gel preparation at the time of application, including consistency, spreadability and moisture content, as well as drug release. The viscosity of the DOX microparticle gels ranged from 36,933 to 46,766 cPs (Table 2). The desired viscosity for gel preparations is in the range of 10,000 - 100,000 cPs (Malik and Kaur, 2018). The viscosity test results show that increasing OA concentration improved the viscosity of the preparation, with all formulations (F1 to F5) within the appropriate range for gel preparations.

Table 2. pH and viscosity of doxycycline microparticle gel preparations with various oleic acid concentrations $(n=3$, mean $\pm S D)$

\begin{tabular}{cccc}
\hline Formulation & $\begin{array}{c}\text { OA } \\
\text { Concentration }\end{array}$ & pH values & Viscosity values \\
\hline F1 & $0 \%$ & $5.91 \pm 0.02$ & $46,766 \pm 1101$ \\
F2 & $2.5 \%$ & $5.86 \pm 0.02$ & $42,233 \pm 1514$ \\
F3 & $5 \%$ & $5.82 \pm 0.02$ & $39,866 \pm 650$ \\
F4 & $7.5 \%$ & $5.73 \pm 0.01$ & $37,866 \pm 650$ \\
F5 & $10 \%$ & $5.52 \pm 0.04$ & $36.933 \pm 152$ \\
\hline
\end{tabular}

\section{Spreadability and in vitro skin occlusivity}

The spreadability test evaluated the ability of the gel preparations to spread and become distributed when applied to the skin. For all formulations, the greater the load applied, the greater the gel spread. The spreadability test results (Table 3) were inversely proportional to the viscosity measurements, with increasing OA concentration reducing spreadability. According to (Nitalikar and Sakarkar, 2013) the spreadability range for semi solid preparations is $3-5 \mathrm{~cm}$ diameter. The spreadability of all the formulations (F1, F2, F3, F4 and F5) was within the desired $3-5 \mathrm{~cm}$ diameter range. 
Evaluation of skin occlusivity was performed in vitro to envisage the ability of the gel formulation to preserve the hydration profile of the skin (Wavikar and Vavia, 2013). Higher gel formulation occlusivity could hypothetically enhance skin hydration for 48 hours, thus permitting drug penetration into the deeper layers of the skin due to a decrease in corneocyte gaps (Purnamawati et al., 2017). The in vitro skin occlusivity results (Table 2) show that the addition of OA increased the occlusivity of all gel formulations. The difference between the OA formulations (F2 to F5) and the control (F1) was statistically significant $(\mathrm{p}<0.05)$.

Table 3. Spreadability and in vitro skin occlusivity of doxycycline (DOX) microparticle gel preparations with various oleic acid concentrations:. $(\mathrm{n}=3$, mean $\pm \mathrm{SD})$

\begin{tabular}{cccc}
\hline Formulation & OA Concentration & $\begin{array}{c}\text { Spreadability } \\
\text { values }(\mathbf{c m})\end{array}$ & $\begin{array}{c}\text { Occlusivity } \\
\text { percentages (\%) }\end{array}$ \\
\hline F1 & $0 \%$ & $3.71 \pm 0.72$ & $84.01 \pm 8.52$ \\
F2 & $2.5 \%$ & $3.98 \pm 0.71$ & $84.93 \pm 8.58$ \\
F3 & $5 \%$ & $4.23 \pm 0.75$ & $86.32 \pm 8.42$ \\
F4 & $7.5 \%$ & $4.59 \pm 0.84$ & $86.35 \pm 8.64$ \\
F5 & $10 \%$ & $4.78 \pm 0.85$ & $87.42 \pm 8.52$ \\
\hline
\end{tabular}

\section{Effect of gel formulation on microparticle size and PdI}

It is important to note that the incorporation of microparticles did not change the characteristics of the microparticles, particularly size and PdI. It was observed the particle size and PDI values of DOX-loaded microparticles were $3.11 \pm 0.24 \mu \mathrm{m}$ and $0.218 \pm 0.03 \mu \mathrm{m}$, respectively. These values were not significantly different $(p>0.05)$ from the initial characteristics of the microparticles. Therefore, the incorporation of the microparticles into the gel formulation did not affect the size and PdI of the DOX-loaded microparticles. This result agreed to previous finding in which it is reported that incorporation of microparticles into gel base did not alter their physical characteristics (Souto et al., 2004).

\section{Ex vivo dermal delivery}

In an attempt to determine the amount of DOX microparticles from the gel preparation that could penetrate through the skin during a certain time interval, ex vivo dermal delivery studies were carried out. The trial results for ex vivo DOX microparticle penetration through the skin (Figure 2a) show that the control (F1) without OA as penetration enhancer was unable to penetrate through the skin after $24 \mathrm{~h}$, as DOX microparticles were unable to penetrate the SC, while increasing the concentration of OA increased ability of DOX microparticles to permeate the skin layer, thus increasing the amount of drug which could be delivered to the site. This role of OA as a penetration enhancing compound acts by disordering the densely arranged stratum corneum intercellular lipid domain. In this study, gel formulation containing DOX without microparticle was prepared and used and control the ex vivo dermal delivery evaluation. The result showed that after $24 \mathrm{~h}, 8739 \pm 783 \mu \mathrm{g}$ of DOX was delivered when DOX was not formulated into microparticles. Therefore, our approach indicated that the formulation of DOX into microparticles could avoid undesired systemic exposure of DOX.

To evaluate the release rate of doxycycline microparticles from the gel preparation, flux calculations (Figure 2b) show that release rate of doxycycline microparticles from the gel increased over time, and was positively associated with OA concentration. The penetration flux can be affected by several factors, one of which is microparticle size. The smaller the particle size, the higher the amount of drug that interacts with the area in SC (Adib et al., 2016). Another is OA concentration, 
with the results confirming that the addition of a suitable concentration of OA to a gel preparation can act as a penetration enhancer and can thereby increase the release rate of an active ingredient.

Permeation data did not fully support the desired local effect because the permeation test was carried out to determine the amount of drug that could penetrate through the skin, whereas to achieve a local effect it is necessary to know the amount of DOX microparticles that could be left on the skin. The purpose of this test was to determine the amount of DOX deposited on the skin. The DOX microparticle retention test (Figure 2c) shows lower amounts of DOX deposited by formulations F1 and F2 (OA concentrations $0 \%$ and $2.5 \%$ ) and were not significantly different from each other ( $\mathrm{p}>$ 0.05 ), indicating that these formulations have a low retention capability in the skin. F3 (5\% OA) was significantly different $(\mathrm{p}<0.05)$ from F4 and F5, with less DOX deposited. The F5 formulation $(10 \%$ OA) had the highest DOX penetration but showed significantly lower DOX retention than F4 (7.5\% OA). This high retention time indicates that F4 could have a longer lasting local effect on the skin. This could be explained by the way in which the PLGA-based polymer microparticle delivery system absorbs medicinal raw materials to heal skin wounds or disease and then controls their release (Han et al., 2016).

Interestingly, F5 formulation which contained the highest (10\%) OA concentration was the most effective in terms of enhanced penetration with the highest rate of dermal delivery, but also had a lower skin retention than F4 (7.5\%), most likely due to the drug swiftly reaching the systemic circulation and thereby reducing the desired local effect (Santoyo and Gartua, 2000) One important goal of the dermal drug delivery system is to maximize the amount of drug that enters the skin layer, thereby increasing the retention time of the drug in the skin. However, several other factors may contribute to the retention profile, including the partition of the drug through the skin layers. (Banning and Heard, 2002) reported that DOX has relatively high affinity to keratin which may attribute to its skin permeation profile as well. In this case, $\mathrm{F} 4$ with $7.5 \% \mathrm{OA}$ was shown to promote enhanced skin permeation, while providing sufficient drug retention in skin layers.

(a)

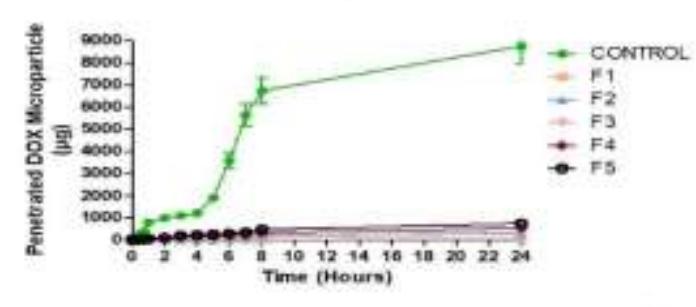

(b)

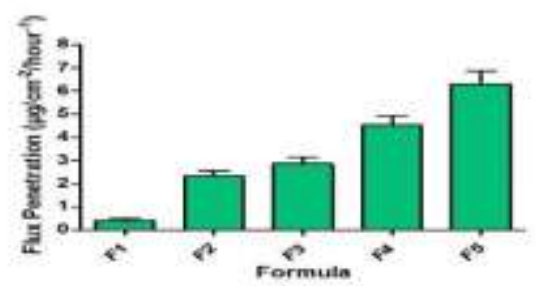

(c)

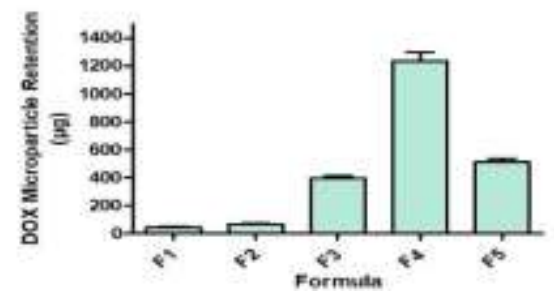

Figure 2. Skin penetration performance of doxycycline (DOX) microparticle gel preparations with various OA concentrations: F1 $(0 \%)$, F2 $(2.5 \%)$, F3 (5\%), F4 (7.5\%) and F5 (10\%): (a) dermal delivery; (b) flux penetration; (c) retention test. Control in the permeation study indicated the formulation without microparticle formulation 


\section{CONCLUSION}

This study demonstrated that varying the concentration of oleic acid (OA) as penetration enhancer in gel preparations affects the physical characteristics, skin penetration, and retention time of a PLGA-based polymer microparticle drug delivery system. The highest OA formulation (10\%) enabled the highest amount of the drug to penetrate the skin, while the highest drug retention was obtained with the $7.5 \%$ OA formulation.

\section{REFERENCES}

Adib, Z. M., Ghanbarzadeh, S., Kouhsoltani, M., Khosroshahi, A. Y., \& Hamishehkar, H. (2016). The effect of particle size on the deposition of solid lipid nanoparticles in different skin layers: A histological study. Advanced Pharmaceutical Bulletin, 6(1), 31-36. https://doi.org/doi.org/10.15171/apb.2016.006

Akalin, G. O., \& Pulat, M. (2018). Preparation and characterization of nanoporous sodium carboxymethyl cellulose hydrogel beads. Journal of Nanomaterials, Vol 2018, Article ID 9676949,1-12. https://doi.org/https://doi.org/10.1155/2018/9676949

Ali, S. M., \& Yosipovitch, G. (2013). Skin pH: From basic science to basic skin care. In Acta Dermato-Venereologica. Acta Derm Venereol, 93(3), 261-267. https://doi.org/https://doi.org/10.2340/00015555-1531

Ardana, M., Aeyni, V., \& Ibrahim, A. (2015). Formulasi dan optimasi basis gel HPMC (Hidroxy Propyl Methyl Cellulose) dengan berbagai variasi konsentrasi. Journal of Tropical Pharmacy and Chemistry, 3(2), 101-108. https://doi.org/https://doi.org/10.25026/jtpc.v3i2.95

Banning, T. P., \& Heard, C. M. (2002). Binding of doxycycline to keratin, melanin and human epidermal tissue. International Journal of Pharmaceutics, 235((1-2)), 219-227. https://doi.org/10.1016/s0378-5173(01)00988-7

Borse, V. A., \& Avinash Balasaheb Gangude Anil Bhavrao Deore. (2020). Formulation and evaluation of antibacterial topical gel of doxycycline hyclate, neem oil and tea tree oil. Indian Journal of Pharmaceutical Education and Research, 54((1)), 206-212. https://doi.org/10.5530/ijper.54.1.24

Campos, E., Branquinho, J., Carreira, A. S., Carvalho, A., Coimbra, P., Ferreira, P., \& Gil, M. H. (2013). Designing polymeric microparticles for biomedical and industrial applications. European Polymer Journal, 49(8), 2005-2021. https://doi.org/10.1016/j.eurpolymj.2013.04.033

Chiller, K., Selkin, B. A., \& Murakawa, G. J. (2001). Skin microflora and bacterial infections of the skin. Journal of Investigative Dermatology Symposium Proceedings, 6((3)), 170-174. https://doi.org/10.1046/j.0022-202x.2001.00043.x

Chukwudi, C. U. (2016). rRNA binding sites and the molecular mechanism of action of the tetracyclines. Antimicrobial Agents and Chemotherapy, 60(8), 4433-4441. https://doi.org/10.1128/AAC.00594-16

Cohen-Sela, E., Chorny, M., Koroukhov, N., \& Golomb, H. D. D. G. (2009). A new double emulsion solvent diffusion technique for encapsulating hydrophilic molecules in PLGA nanoparticles. Journal of Controlled Release, 133 (2), 90-95. https://doi.org/10.1016/j.jconrel.2008.09.073

Nandini, D,. NS, Chauhan., \& K, Chandra. A., Pathak. (2009). Effect of permeation enhancers on the release and permeation kinetics of oxytetracycline hydrochloride organogel formulations. Journal of Young Pharmacists, 1((4)), 296-300. https://doi.org/10.4103/0975-1483.59314

Dian Permana, A., Nurul Utami, R., J.Courtenay, A., A.Manggau, M., F.Donnelly, R., \& Rahman, L. (2020). Phytosomal nanocarriers as platforms for improved delivery of natural antioxidant and photoprotective compounds in propolis: An approach for enhanced both dissolution behaviour in biorelevant media and skin retention profiles. Journal of Photochemistry and Photobiology B: Biology, 205(111846). https://doi.org/10.1016/j.jphotobiol.2020.111846

Elisa Campos, J.Branquinho, S.Carreira, A., Carvalho, A., Coimbra, P., \& P.FerreiraM.H.Gil. (2013). Designing polymeric microparticles for biomedical and industrial applications. European Polymer Journal, 49((8)), 2005-2021. https://doi.org/10.1016/j.eurpolymj.2013.04.033 
Han, F. Y., Thurecht, K. J., Whittaker, A. K., \& Smith, M. T. (2016). Bioerodable PLGA-based microparticles for producing sustained-release drug formulations and strategies for improving drug loading. Frontiers in Pharmacology, 7(185), 1-11. https://doi.org/10.3389/fphar.2016.00185

J.Hatlen, T., \& G.Miller, L. (2021). Staphylococcal skin and soft tissue infections. Infectious Disease Clinics of North America, 35(1), 81-105. https://doi.org/10.1016/j.idc.2020.10.003

Kalan, C., \& Femling, J. (2017). Skin and soft tissue infections: causes and treatments. Physician Assistant Clinics, 2(3), 421-433. https://doi.org/10.1016/j.cpha.2017.02.006

Kim, Y., Beck-Broichsitter, M., \& Banga, A. K. (2019). Design and evaluation of a poly(Lactide-coglycolide)-based in situ film-forming system for topical delivery of trolamine salicylate. Pharmaceutics, 11(8), 1-14. https://doi.org/10.3390/pharmaceutics11080409

Lengyel, M., Kállai-Szabó, N., Antal, V., Laki, A. J., \& Antal, I. (2019). Microparticles, microspheres, and microcapsules for advanced drug delivery. Scientia Pharmaceutica, 87(3), 1-31. https://doi.org/10.3390/scipharm87030020

Lopez, C. G., Colby, R. H., \& Cabral, J. T. (2018). Electrostatic and hydrophobic interactions in NaCMC aqueous solutions: effect of degree of substitution. Polyelectrolyte Structure, Dynamics and Solution Rheology, 1-22. https://doi.org/10.1021/acs.macromol.8b00178

Machado, H. A., Abercrombie, J. J., You, T., DeLuca, P. P., \& Leung, K. P. (2013). Release of a wound-healing agent from PLGA microspheres in a thermosensitive gel. BioMed Research International, Article ID 387863, 1-11. https://doi.org/10.1155/2013/387863

Marco, D. B. A. De, Giuffrida, R., Conforti, C., Barlusconi, C., Foti, C., \& Romita, P. (2020). Topical antibiotics in the dermatological clinical practice: Indications, efficacy, and adverse effects. Dermatologic Therapy, 33((6):e13824). https://doi.org/10.1111/dth.13824

Mazzara, J. M., Ochyl, L. J., Hong, J. K. Y., Moon, J. J., Prausnitz, M. R., \& Schwendeman P, S. (2019). Self-healing encapsulation and controlled release of vaccine antigens from PLGA microparticles delivered by microneedle patches. Bioeng Transl Med, 4((1)), 116-128. https://doi.org/10.1002/btm2.10103

Nitalikar, M. M., \& Sakarkar, D. M. (2013). Formulation and evaluation of topical gel of Meloxicam with Beta-Cyclodextrin complex. Research Journal of Pharmacy and Technology, 6((7)), 790793

Patil, P. B., Datir, S. K., \& Saudagar, R. B. (2019). A review on topical gels as drug delivery system. Journal of Drug Delivery and Therapeutics, 9(3-(s)), 989-994. https://doi.org//10.22270/jddt.v9i3-s.2930

Permana, A. D., Mir, M., Utomo, E., \& F.Donnelly, R. (2020). Bacterially sensitive nanoparticlebased dissolving microneedles of doxycycline for enhanced treatment of bacterial biofilm skin infection: A proof of concept study. International Journal of Pharmaceutics: X, 2(100047). https://doi.org/10.1016/j.ijpx.2020.100047

Prakash, D., Singh, A. P., Katiyar, N. S., \& Pathak, K. (2016). Penetration enhancers: adjuvants in transdermal drug delivery system. World Journal of Pharmacy and Pharmaceutical Sciences, 5((5)), 353-376. https://doi.org/10.20959/wjpps20165-6654

Prow, T. W., Grice, J. E., Lin, L. L., Faye, R., Butler, M., Becker, W., ... Roberts, M. S. (2011). Nanoparticles and microparticles for skin drug delivery. Advanced Drug Delivery Reviews, 63(6), 470-491. https://doi.org/10.1016/j.addr.2011.01.012

Purnamawati, S., Indrastuti, N., Danarti, R., \& Saefudin, T. (2017). The role of moisturizers in addressing various kinds of dermatitis: A review. In Clinical Medicine and Research , 15( 3), 75-87. Marshfield Clinic. https://doi.org/10.3121/cmr.2017.1363

Santoyo, S., \& Gartua, P. Y. (2000). Effect of skin pretreatment with fatty acids on percutaneous absorption and skin retention of piroxicam after its topical application. European Journal of Pharmaceutics and Biopharmaceutics, 50(2), 245-250. https://doi.org/10.1016/S09396411(00)00097-7 
Singh Malik, D., \& Kaur, G. (2018). Nanostructured gel for topical delivery of azelaic acid: Designing, characterization, and in-vitro evaluation. Journal of Drug Delivery Science and Technology, 47, 123-136. https://doi.org/10.1016/j.jddst.2018.07.008

Smith, R., Russo, J., Fiegel, J., \& Brogden, N. (2020). Antibiotic delivery strategies to treat skin infections when innate antimicrobial defense fails. Antibiotics, 9(2), 56, 1-25. https://doi.org/10.3390/antibiotics9020056

Soomherun, N., Kreua-ongarjnukool, N., Chumnanvej, S., \& Thumsing, S. (2017). Encapsulation of nicardipine hydrochloride and release from biodegradable poly(D,L-lactic-co-glycolic acid) microparticles by double emulsion process: effect of emulsion stability and different parameters on drug entrapment. International Journal of Biomaterials, Article ID, 1-13. https://doi.org/10.1155/2017/1743765

Souto, E. B., Wissing, S. A., Barbosa, C. M., \& Müller, R. H. (2004). Evaluation of the physical stability of SLN and NLC before and after incorporation into hydrogel formulations. European Journal of Pharmaceutics and Biopharmaceutics, 58((1)), 83-90. https://doi.org/10.1016/j.ejpb.2004.02.015

Wavikar, P., \& Vavia, P. (2013). Nanolipidgel for enhanced skin deposition and improved antifungal activity. AAPS PharmSciTech, 14((1)), 222-233. https://doi.org/10.1208/s12249-012-9908-y

Wohlrab, J., \& Gebert, A. (2018). PH and Buffer Capacity of Topical Formulations. Current Problems in Dermatology (Switzerland), 54, 123-131. https://doi.org/10.1159/000489526 
